\title{
RESEARCH
}

Open Access

\section{Comparison of senescence-related changes between three- and two-dimensional cultured adipose-derived mesenchymal stem cells}

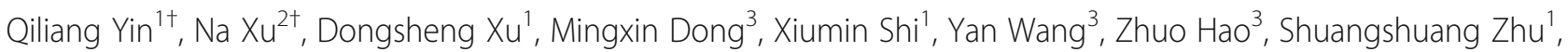
Donghai Zhao ${ }^{2}$, Haofan Jin $^{1 *}$ and Wensen Liü ${ }^{3^{*}}$

\begin{abstract}
Background: Adipose-derived mesenchymal stem cells (ADMSCs) have attracted widespread interest as cell-based tissue repair systems. To obtain adequate quantities of ADMSCs for therapeutic applications, extensive in vitro expansion is required. However, under current two-dimensional (2D) approaches, ADMSCs rapidly undergo replicative senescence, and cell growth is impeded and stem cell properties are eliminated by mechanisms that are poorly understood. These issues limit the extensive applications of ADMSCs. In this study, we investigated senescence-related changes in mesenchymal stem cells (MSCs) isolated from human adipose tissue in 2D and three-dimensional (3D) cultures.

Methods: We studied cell growth over a given period (21 days) to determine if modes of culture were associated with ADMSC senescence. ADMSCs were isolated from healthy females by liposuction surgery and then were grown in 2D and $3 \mathrm{D}$ cultures. The cell morphology was observed during cell culture. Every other time of culture, senescence-associated $\beta$-galactosidase (SA- $\beta$-gal) expression, cell viability, proliferation, and differentiation potential of ADMSCs from 2D and 3D cultures were detected. Also, senescence- and stemness-related gene expression, telomere length, telomerase activity, and energy metabolism of ADMSCs for different culture times were evaluated.

Results: With long-term propagation, we observed significant changes in cell morphology, proliferation, differentiation abilities, and energy metabolism, which were associated with increases in SA- $\beta$-gal activity and decreases in telomere length and telomerase activity. Notably, when cultured in 3D, these changes were improved. Conclusions: Our results indicate that 3D culture is able to ameliorate senescence-related changes in ADMSCs. Keywords: Adipose-derived mesenchymal stem cells, Three-dimensional culture, Senescence, Energy metabolism
\end{abstract}

\footnotetext{
*Correspondence: kinhf1968@126.com; liuws85952@163.com

${ }^{\dagger}$ Qiliang Yin and $\mathrm{Na}$ Xu contributed equally to this work.

${ }^{1}$ Cancer Center at the First Hospital of Jilin University, 1 Xinmin Street,

Changchun 130021, People's Republic of China

${ }^{3}$ Institute of Military Veterinary Medicine, Academy of Military Medical

Sciences, Zoonosis Prevention and Control Key Laboratory, Changchun

130122, People's Republic of China

Full list of author information is available at the end of the article
}

\section{$\triangle B M C$}

(c) The Author(s). 2020 Open Access This article is licensed under a Creative Commons Attribution 4.0 International License, which permits use, sharing, adaptation, distribution and reproduction in any medium or format, as long as you give appropriate credit to the original author(s) and the source, provide a link to the Creative Commons licence, and indicate if changes were made. The images or other third party material in this article are included in the article's Creative Commons licence, unless indicated otherwise in a credit line to the material. If material is not included in the article's Creative Commons licence and your intended use is not permitted by statutory regulation or exceeds the permitted use, you will need to obtain permission directly from the copyright holder. To view a copy of this licence, visit http://creativecommons.org/licenses/by/4.0/ The Creative Commons Public Domain Dedication waiver (http://creativecommons.org/publicdomain/zero/1.0/) applies to the data made available in this article, unless otherwise stated in a credit line to the data. 


\section{Background}

Mesenchymal stem cells (MSCs) are pluripotent stem cells with the potential to self-replicate and multidifferentiate [1, 2]. Adipose tissue is an important source of MSCs [3]. Adipose-derived mesenchymal stem cells (ADMSCs) are harvested with low donor-site morbidity and are not associated with ethical issues; therefore, they represent promising candidates for various clinical applications, including tissue repair and regenerative medicine $[2,4]$. In recent years, ADMSCs have been shown to promote revascularization, activate local stem cell niches, reduce oxidative stress, and modulate immune responses [3, 5]. However, therapies utilizing ADMSCs often require ex vivo expansion to generate large quantities of cells required for patients $[6,7]$. Therefore, in vitro expansion is particularly important for ADMSCs.

Two-dimensional (2D) culture has been routinely used over the past several decades [8]. Typically, MSCs are expanded on stiff tissue culture-treated polystyrene, as a 2D monolayer [6]. However, growing cells in flat layers on plastic surfaces do not accurately mimic the natural in vivo cellular microenvironment, due to a lack of three-dimensional cues from the external media [9]. Moreover, the cellular microenvironment can seriously influence MSC characteristics and cause issues when transferring basic research to clinical settings [10]. Accumulating evidence indicates that extensive passaging of ADMSCs in 2D culture induces replicative senescence, resulting in cell cycle arrest, cell morphology and metabolic changes, and loss of differentiation potential [1114]. Therefore, ADMSCs are prone to senescence and are difficult to maintain during long-term 2D expansion [15]. As a result, efforts have led to the development of novel approaches to recreate more physiologically relevant environments in the form of three-dimensional (3D) culture $[16,17]$.

$3 \mathrm{D}$ culture is a simple and effective culture system, developed on the basis of a $2 \mathrm{D}$ monolayer, but with in vivo animal model characteristics [18, 19]. Currently, 3D culture systems are gaining interest with regard to recreating complex extracellular microenvironments (ECMs), thereby providing insights into conditions experienced by MSCs. When compared to 2D approaches, 3D culture creates an artificial ECM where cells grow or interact with their surroundings in three dimensions $[8,20]$. In such cultures, there is increased MSC communication with neighboring cells, and cell-to-cell and cell-to-matrix connections are easily formed [21]. Reports from the literature suggest that 3D culture of human umbilical cord MSCs promotes cell yields, maintains stemness, and represents a promising strategy for cell expansion on industrial levels, with great potential for cell therapy and biotechnology [22-24]. Additionally, 3D microenvironments encourage
MSC growth and differentiation into hepatocyte-like cells, in the presence of growth factors [25]. More importantly, the utilization of $3 \mathrm{D}$ culture techniques circumvents issues surrounding altered cellular properties of extensively expanded MSCs [7, 21]. In this work, we focus on the benefits of using 3D cultures and investigate ideal and optimized living environments for ADMSC growth in vitro.

\section{Methods}

\section{Isolation and cultivation of human ADMSCs}

Abdominal adipose tissue was obtained from five healthy females by liposuction surgery. Lipoaspirate MSCs were isolated and characterized as previously described [26]. Cells in culture were maintained at $37^{\circ} \mathrm{C}$ with $5 \% \mathrm{CO}_{2}$, until $80 \%$ cell confluence; then, cells were passaged on. ADMSCs from the third passage were plated in conventional 2D or 3D culture vessels. For 2D culture, cells from the third passage were cultured in six-well plates at $1 \times 10^{6}$ cells $/ \mathrm{ml}$. Hydrogel (TheWell Bioscience, catalog no. TWG002, Shanghai, China), as a non-animal-derived polysaccharide hydrogel system that mimics the natural cellular microenvironment, is a new synthetic biomaterial for cell expansion in vitro and is the main constituent of 3D culture. The hydrogel was mixed with cell culture medium to form a hydrogel matrix. The dilution ratio was $1: 1$, i.e., hydrogel to PBS, $1: 1$, v/v. The hydrogel and ADMSCs were uniformly mixed and were seeded into a six-well plate at $1 \times 10^{6}$ cells $/ \mathrm{ml}$ as the $3 \mathrm{D}$ culture. Then, the cell culture medium (DAKEWE, EliteGro ${ }^{\mathrm{Tm}}$ Adv, Beijing, China) was added to cover the hydrogel carefully. The medium was replaced every 3 days. When ADMSCs grew to $80 \%$ confluence, the cells were passaged at 1:2. It was a little different for the cell passage of 3D culture. Firstly, preheated $0.1 \times$ PBS and empty centrifuge tube in $37^{\circ} \mathrm{C}$ water bath before taking out the cell culture plate from incubator. Discarded the medium covered with the top of the hydrogel, added $1 \mathrm{ml}$ of preheated $0.1 \times$ PBS into each hole, and thoroughly mixed. Then, put the mixture into the preheated centrifuge tube and rinsed each hole with $1 \mathrm{ml}$ preheated $0.1 \times \mathrm{PBS}$, and added preheated $0.1 \times$ PBS to $10 \mathrm{ml}$, thoroughly mixed. Finally, collected the cells after centrifuging at $1000 \mathrm{rpm}$ for $5 \mathrm{~min}$. ADMSCs were reseeded at six-well plate.

The first day (d 1) was defined when ADMSCs from the third passage were seeded into six-well plates, as described.

\section{Characterization of ADMSCs}

Flow cytometry assessment was conducted to confirm the mesenchymal origin of cells. Third passage cells were resuspended following digestion with $0.125 \%$ trypsin. A minimum of $1 \times 10^{5}$ cells $/ \mathrm{ml}$ were collected from six-well plates. Rat monoclonal anti-human antibodies 
were used at a dilution of 1:150 for all cell surface markers. MSCs were incubated with phycoerythrin (PE)coupled antibodies, CD34 (sc-7324; Santa Cruz) and CD45 (554,878; BD Biosciences), and fluorescein isothiocyanate (FITC)-coupled antibodies, CD44 (550,974; BD Biosciences) and CD105 (BD Biosciences), in the dark at room temperature for $30 \mathrm{~min}$. IgG1-PE and IgG1-FITC were used as isotype controls. Cell data were analyzed using the Paint-A-Gate Pro ${ }^{\text {tw }}$ software.

Senescence-associated (SA)- $\beta$-galactosidase (gal) assay ADMSCs from 2D and 3D cultures at 3 days, 7 days, 14 days, and 21 days were seeded in six-well plates at $1 \times 10^{5}$ cells/well overnight. The next day, cells were fixed for $30 \mathrm{~min}$ at room temperature in $4 \%$ formaldehyde and washed twice in PBS (pH 7.3). Then, ADMSCs were incubated overnight at $37^{\circ} \mathrm{C}$ with freshly prepared SA- $\beta$-gal stain solution (C0602, Beyotime, Shanghai, China) following the manufacturer's instructions. At least 400 cells were observed in randomly chosen, nonoverlapping fields by three independent observers to quantify SA- $\beta$-gal expression. Positive cells were stained blue and counted in three randomly selected fields under the microscope (XPF-550C, caikon, Shanghai, China). The experiment was performed three times, and the mean percentage of cells expressing SA- $\beta$-gal was calculated.

\section{Cell viability}

ADMSC viability was determined using Muse Count \& Viability Assay in the Muse Cell Analyzer (Luminex, USA) according to the manufacturer's instructions. Cells $\left(1 \times 10^{6}\right.$ cells/well $)$ were harvested from both $2 \mathrm{D}$ and $3 \mathrm{D}$ cultures. They were then resuspended in medium to achieve a cell density of $2 \times 10^{5}$ cells $/ \mathrm{ml}$, from which $50 \mu \mathrm{l}$ cells and $450 \mu \mathrm{l}$ Muse ${ }^{\mathrm{Tw}}$ Count \& Viability reagent were added to a tube, and incubated for $5 \mathrm{~min}$ in the dark at room temperature. Cell viability was evaluated by miniaturized fluorescence detection and microcapillary cytometry with Muse Cell Analyzer. In addition, we counted the cells in 2D and 3D cultures at every measured time point by Muse Cell Analyzer.

\section{Stain of living cells}

ADMSCs from 2D and 3D cultures at 3 days, 7 days, 14 days, and 21 days were stained by HCS NuclearMask $^{\text {ma }}$ Blue Stain (H10325, Invitrogen, Shanghai, China) according to the manufacturer's instructions. The cells were pulsed with $100 \mu \mathrm{l}$ staining solution (1:2000) for 30 min at room temperature. After the incubation, the stained cells were analyzed by fluorescence microscopy (IX71, OLYMPUS, Xiameng, China).

\section{Adipo- and osteogenic differentiation of ADMSCs}

ADMSCs from 3 days, 7 days, 14 days, and 21 days were induced to undergo adipogenic and osteogenic differentiation, to identify cell capacity for differentiation in 2D and 3D cultures. For adipogenic differentiation, ADMSCs were seeded in 24-well plates at a density of $1 \times 10^{5}$ cells/well. MSCgo $^{\text {Th }}$ adipogenic differentiation medium (catalog no. 05412, STEMCELL, USA) was used to induce adipogenic differentiation after cells reached 95\% confluence. Induction/maintenance media were replaced for cycles of 3 days/1 day, respectively. Differentiated cells were assessed by staining intracellular lipid droplets with Oil Red O (MC37A0-1.4, VivaCell BIOSCIENCES, Shanghai, China) after 21 days in adipogenesis induction medium. For osteogenic differentiation, a similar process was adopted. $\mathrm{MSCgo}^{\mathrm{ma}}$ osteogenic differentiation medium (catalog no. 05465, STEMCELL, USA) induced osteogenic differentiation when cells reached 95\% confluence. Differentiated cells were stained using Alizarin Red S (MC37C0-1.4, VivaCell BIOSCIENCES, Shanghai, China) staining of accumulated calcium deposits, after 28 days of differentiation. At least 300 cells were observed in randomly chosen, non-overlapping fields by three independent observers to quantify cell differentiation capacity. Positive cells were counted in three randomly selected fields under the microscope (XPF550C, caikon, Shanghai, China).

\section{Real-time fluorescence quantitative polymerase chain reaction (RT-qPCR)}

Total RNA was isolated from ADMSCs using TRIzol reagent (Invitrogen, USA) following the manufacturer's instructions. cDNA was prepared by reverse transcription using the PrimeScript ${ }^{\text {tw }}$ RT-PCR Kit (TaKaRa, Japan). Next, mRNA levels were quantified for aging-related genes (p16, p21, p53) and stemness-related genes (Sox2, Oct4, Nanog, c-myc) by RT-qPCR on an ABI Prism7900 Detector (Applied Biosystems, USA), using SYBR Premix Ex $\operatorname{Taq}^{\mathrm{Tm}} \cdot \beta$-actin was used as a reference gene. Each experimental group was analyzed in triplicate. mRNA expression was calculated using the $2^{-\Delta \Delta \mathrm{Ct}}$ method. Primer sequences for RT-qPCR are shown in Table 1.

\section{Telomere length and activity assay}

Genomic DNA (gDNA) was isolated from ADMSCs and used as a template for RT-qPCR. The relative telomere length of ADMSCs from 3 days, 7 days, 14 days, and 21 days was assayed using the relative human telomere length quantification RT-qPCR assay kit (catalog no. 8908, ScienCell, USA) following the manufacturer's instructions. Data were analyzed using the comparative quantification cycle value $(\Delta \Delta \mathrm{Cq})$ method. Telomeres are maintained by telomerase, which comprises telomerase reverse transcriptase (TERT) and telomerase RNA 
Table 1 Primers used for RT-qPCR

\begin{tabular}{|c|c|c|}
\hline Gene & Primer sequence $\left(5^{\prime}-3^{\prime}\right)$ & Product size $(b p)$ \\
\hline \multirow[t]{2}{*}{$\beta$-actin } & Forward: CATGTACGTTGCTATCCAGGC & 250 \\
\hline & Reverse: CTCCTTAATGTCACGCACGAT & \\
\hline \multirow[t]{2}{*}{ P16 } & Forward: ATCATCAGTCACCGAAGG & 369 \\
\hline & Reverse: TCAAGAGAAGCCAGTAACC & \\
\hline \multirow[t]{2}{*}{ P21 } & Forward: CATCTTCTGCCTTAGTCTCA & 163 \\
\hline & Reverse: CACTCTTAGGAACCTCTCATT & \\
\hline \multirow[t]{2}{*}{ P53 } & Forward: CGGACGATATTGAACAATGG & 158 \\
\hline & Reverse: GGAAGGGACAGAAGATGAC & \\
\hline \multirow[t]{2}{*}{ SOX2 } & Forward: GCCGAGTGGAAACTITTTCG & 155 \\
\hline & Reverse: GGCAGCGTGTACTTATCCTTCT & \\
\hline \multirow[t]{2}{*}{ OCT4 } & Forward: CTGGGTTGATCCTCGGACCT & 243 \\
\hline & Reverse: CCATCGGAGTTGCTCTCCA & \\
\hline \multirow[t]{2}{*}{ Nanog } & Forward: TCTATAACTGTGGAGAGGAATC & 122 \\
\hline & Reverse: GGTCTGCTGTATTACATTAAGG & \\
\hline \multirow[t]{2}{*}{ c-myc } & Forward: GTCAAGAGGCGAACACACAAC & 162 \\
\hline & Reverse: TTGGACGGACAGGATGTATGC & \\
\hline \multirow[t]{2}{*}{ TERT } & Forward: AAATGCGGCCCCTGTTTCT & 76 \\
\hline & Reverse: CAGTGCGTCTTGAGGAGCA & \\
\hline
\end{tabular}

component (TERC) [27]; thus, TERT expression is consistent with telomerase activity. The RT-qPCR assay indirectly reflects telomerase activity by detecting TERT mRNA expression levels. The procedure was identical to the above.

\section{Relative mitochondrial DNA copy number quantification} Mitochondrial DNA (mtDNA) from ADMSCs was used as a template for RT-qPCR. The relative mtDNA copy number of ADMSCs from different time points was determined using the relative human mtDNA copy number quantification RT-qPCR assay kit (catalog no. 8938, ScienCell, USA) following the manufacturer's instructions. Data were analyzed using the comparative $\Delta \Delta \mathrm{Cq}$ method.

\section{Cellular energy metabolism studies}

The extracellular acidification rate (ECAR) and oxygen consumption rate (OCR) of cells were detected using the XF96 extracellular flux analyzer (Seahorse Bioscience; North Billerica, MA, USA) following the manufacturer's instructions. ADMSCs were seeded at $5 \times 10^{3}$ cells/well in a 96-well Seahorse culture plate (Seahorse Bioscience, North Billerica, MA, USA), before conducting the experiment. For the ECAR assay, studies were performed in un-buffered DMEM (catalog no. 11965092, Gibco ${ }^{\text {Tm }}$, USA), pH 7.3 at $37^{\circ} \mathrm{C}$. Glucose $(8 \mathrm{mM})$, oligomycin A (oligo; an ATP synthase inhibitor, $0.8 \mu \mathrm{M}$ ), and 2deoxyglucose (2-DG; inhibitor of glycolysis; $80 \mathrm{mM}$ ) were added to different ports of the Seahorse cartridge.
For OCR assays, analyses were conducted in a medium consisting of $20 \mathrm{mM}$ glucose and $1.8 \mathrm{mM}$ sodium pyruvate in un-buffered DMEM, pH 7.3, at $37^{\circ} \mathrm{C}$. Oligomycin A $(1 \mu \mathrm{M})$, carbonyl cyanide m-chlorophenylhydrazone (FCCP; a mitochondrial uncoupler; $400 \mathrm{nM}$ ), rotenone (complex I inhibitor; $0.8 \mu \mathrm{M}$ ), and antimycin A (complex III inhibitor; $0.8 \mu \mathrm{M}$ ) were added to different ports of the Seahorse cartridge. Each experimental group was assayed with four to five replicates in each analysis. ECAR and OCR data were normalized to cell numbers, as detected by CellTiter-Glo analysis (Promega, USA) at assay end.

\section{Statistical data analysis}

The numbers of repeats were at least 3 times for each experiment at both technical repeats and biological replicates. Numerical data were reported using means \pm standard deviation (SD). Data analyses were performed using paired $t$ tests with the GraphPad Prism 7 software. Statistical differences were assessed at $p<0.05, p<0.01$, and $p<0.001$.

\section{Results \\ Morphological characteristics of ADMSCs}

Primary ADMSCs from culture are shown in Additional file 1: Fig. S1. These cells exhibited fibroblast-like, spindle-shaped morphology; were spiral-shaped; and were in alignment.

ADMSCs from the third passage were characterized by flow cytometry, indicating the presence of CD34- and CD45-negative $(0.89 \%)$ surface markers (Additional file 2: Fig. S2a) and CD44- and CD105-positive (99.49\%) surface markers (Additional file 2: Fig. S2b).

As described, ADMSCs from the third passage were plated in 2D and 3D cultures (Additional file 3: Fig. S3) and photographed at 3 days, 7 days, 14 days, and 21 days (Fig. 1). Cell morphology varied with different time points and culture modes. In 2D culture, cells showed a fibroblast-like morphology, were spindle-shaped, and were in alignment. At 3 days and 7 days, they were relatively homogeneous; cells had the characteristic spindle shape and the cell surface appeared smooth (Fig. 1a, b). At 14 days, 2D cultured cells still maintained the characteristic MSC shape; however, some cells displayed pseudopod-like structures, i.e., they were longer and flatter (Fig. 1c). Unlike 3 days and 7 days, cell shape at 21 days was flat, and almost all ADMSCs had lost their MSC shape, i.e., cells were focally aggregated and exhibited a "fried egg" morphology (Fig. 1d).

In contrast, there were no morphological variations in $3 \mathrm{D}$ culture, ADMSCs grew in a hydrogel suspension, and most cells were round in shape, with a gradually decreasing cell density in relation to time points (Fig. 1e-h).

In addition, ADMSCs were retrieved and re-cultured in six-well plates, without hydrogel after 3D culture for 


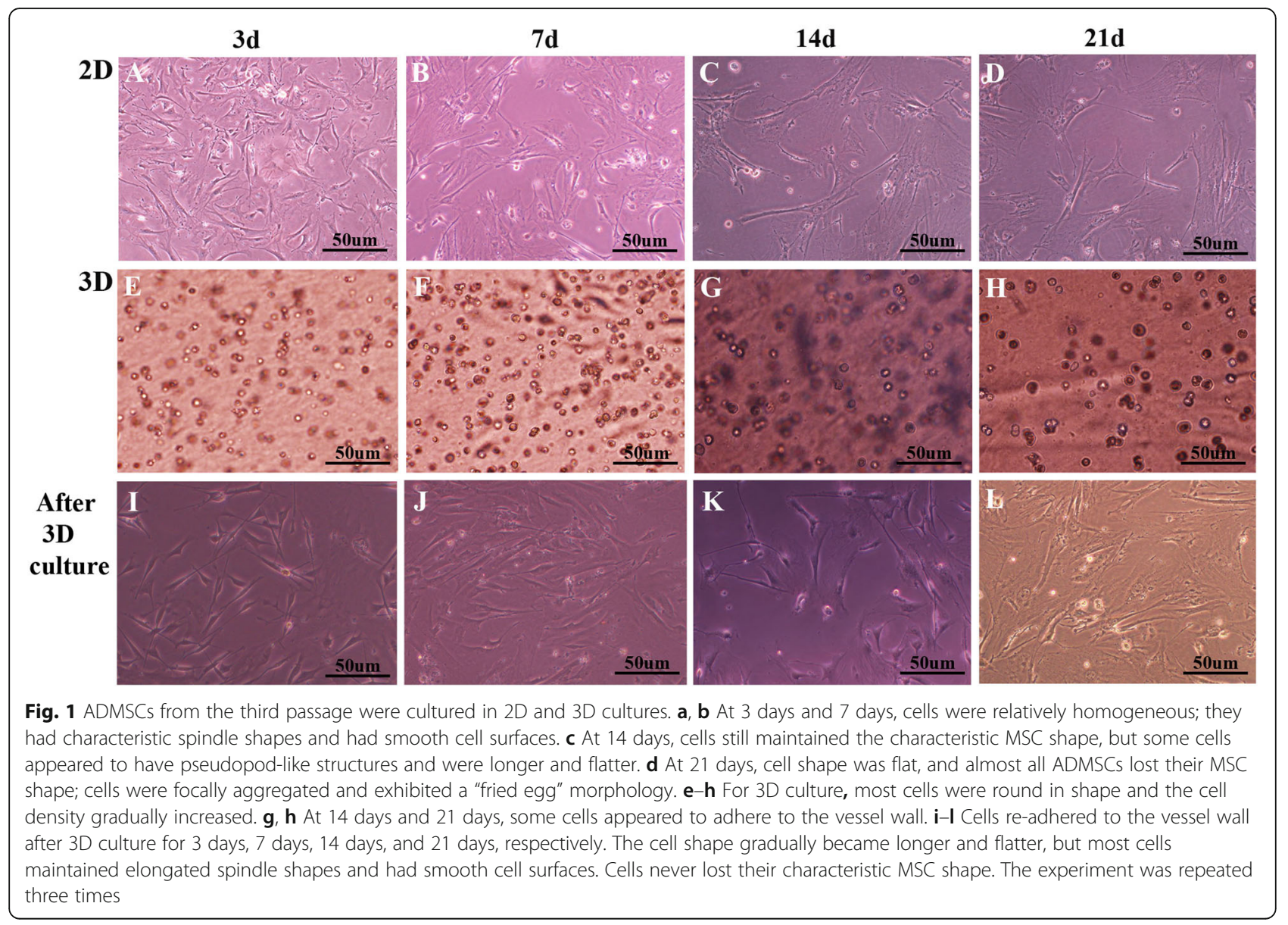

3 days, 7 days, 14 days, and 21 days. Although cell shape gradually became longer and flatter as time progressed, most cells maintained elongated spindle shapes and smooth cell surfaces. More importantly, cells never lost their characteristic MSC shape (Fig. 1i-l).

\section{Evaluating senescence-associated (SA) $\beta$-galactosidase (gal) expression}

ADMSCs from $2 \mathrm{D}$ and $3 \mathrm{D}$ cultures were stained with SA- $\beta$ gal. Aging cells stained blue reflected SA- $\beta$-gal expression. As anticipated, little or no expression was observed at 3 days and 7 days in $2 \mathrm{D}$ and 3D cultures, but subsequent increases in expression were observed with cultivation times. While cell senescence was more visible in $2 \mathrm{D}$ cells when compared with 3D cells at 14 days and 21 days (Fig. 2a), blue ADMSCs in $2 \mathrm{D}$ culture were significantly increased when compared to $3 \mathrm{D}$ culture at 14 days $(-9.417 \pm 0.651)$. Strikingly, most ADMSCs were stained blue at 21 days in 2D cultures, but limited cell densities were stained blue in 3D culture (26.08 \pm 0.363 ), at the same time period (Fig. $2 \mathrm{~b}$ ).

\section{ADMSC viability}

ADMSC viability was assessed in $2 \mathrm{D}$ and $3 \mathrm{D}$ cultures at different time points (Fig. 3a). As expected, ADMSC viability was comparable at 3 days and 7 days under both culture conditions, and the highest viability appeared at 7 days. However, viability decrease was observed at 14 days and 21 days and was significantly decreased in $2 \mathrm{D}$ cultures when compared with 3D. Significantly, cell viability in 3D culture was greater than in 2D cultures at 14 days $(7.933 \pm 1.281)$ and 21 days $(6.133 \pm 1.255)$ (Fig. 3b). Meanwhile, ADMSC number in 2D and 3D cultures at every measured time point was counted (Fig. 3c). Cell number was significantly increased within 7 days in $2 \mathrm{D}$ and $3 \mathrm{D}$ cultures, and the population doubling time was 6 days and 4 days, respectively; subsequently, the number of cells decreased rapidly, especially in 2D culture. Obviously, the cell number in 3D cultures was significantly greater than that in $2 \mathrm{D}(0.586 \pm 0.411)$. Additionally, analysis of ADMSC staining showed that the number of living cells in 2D and 3D cultures was basically in accordance with the result of cell viability (Fig. 3d).

\section{Adipo- and osteogenic differentiation of ADMSCs}

Differences in adipo- and osteogenic capacities of ADMSCs in 2D and 3D cultures over the time points 3 days, 7 days, 14 days, and 21 days were analyzed (Fig. 3e, f). Whether in 2D or 3D culture, the adipo- and 


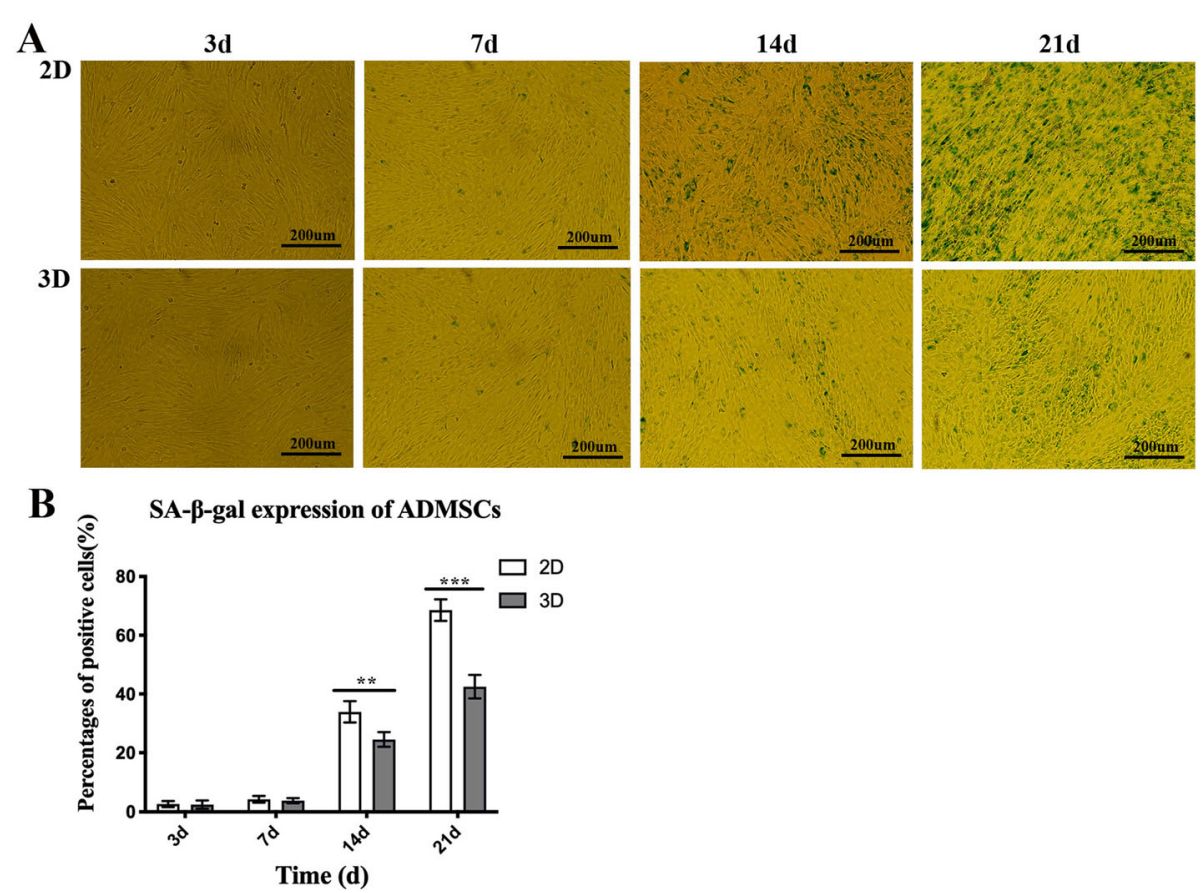

Fig. 2 The expression of senescence-associated $\beta$-galactosidase in 2D and 3D ADMSC cultures. a, $\mathbf{b}$ Little or no SA- $\beta$-gal expression was detected at 3 days and 7 days in either cultures, but increased expression was observed during progressive cultivation with time. Blue ADMSCs in 2D culture were statistically higher than 3D cultures at 14 days. At 21 days, most ADMSCs were stained blue in 2D culture, but limited cells were stained blue in 3D culture at the same time period. Data represented as average \pm SD from $n=3$ experiments, ${ }^{* *} p<0.01,{ }^{* * *} p<0.001$

osteogenic differentiation potential of ADMSCs were similar at 3 days and 7 days. However, an obvious agerelated decline was observed in adipo- and osteogenic differentiation capacity for ADMSCs at 14 days (13.58 \pm 2.184 and $12.75 \pm 3.031$, respectively) and 21 days $(11.58 \pm 2.309$ and $10.95 \pm 2.282$, respectively) (Fig. $3 g$, h) in 2D cultures. Notably, in this culture, ADMSCs nearly lost osteogenic differentiation at 21 days (Fig. 3h).

\section{Evaluating aging- and stemness-related gene expression} Changes in aging- and stemness-related gene expression were evaluated by quantifying mRNA levels in ADMSCs from both culture conditions. As expected, the expression of age-related genes p16, p21, and p53 was gradually increased with culture time; furthermore, gene expression in ADMSCs from 2D culture were significantly higher than those from $3 \mathrm{D}$ cultures at 14 days ($0.767 \pm 0.169,-0.559 \pm 0.019$, and $0.351 \pm 0.023$, respectively) and 21 days $(-1.009 \pm 0.178,-0.975 \pm 0.014$, and $-0.947 \pm 0.203$, respectively). Conversely, the expression levels of stemness-related genes, Sox2, Oct4, Nanog, and c-myc, exhibited a decreasing tendency with long-term expansion. Moreover, Oct4 and Nanog expression exhibited a more significant reduction in $2 \mathrm{D}$ culture than in $3 \mathrm{D}$ cultures at 14 days $(0.998 \pm 0.021$ and $0.921 \pm 0.068$, respectively) and 21 days $(0.695 \pm 0.201$ and $0.997 \pm$ 0.307 , respectively); besides, Sox 2 and c-myc expression were significantly decreased in $2 \mathrm{D}$ cultures at 21 days (0.693 \pm 0.125 and $0.616 \pm 0.143$, respectively) (Fig. 4).

\section{Evaluation of relative telomere length, telomerase} activity, and relative mitochondrial DNA copy number We identified that telomere length in ADMSCs gradually shortened during cell expansion in both 2D and $3 \mathrm{D}$ cultures. However, telomere length shortened significantly more in $2 \mathrm{D}$ culture when compared to $3 \mathrm{D}$, especially for 14 days $(0.274 \pm 0.047)$ and 21 days $(0.223 \pm$ 0.0052). It seemed that the decline fluctuation of telomere length was steadier in 3D culture (Fig. 5a). In addition, TERT expression revealed that changes in telomerase activity agreed with relative telomere lengths in ADMSCs (Fig. 5b). Similarly, relative mtDNA copy numbers of ADMSCs in 2D and 3D cultures were gradually decreased, but mtDNA copy numbers in 3D culture were significantly higher when compared with $2 \mathrm{D}$ cultures at 14 days $(0.315 \pm 0.043)$ and 21 days $(0.349 \pm$ 0.055) (Fig. 5c).

\section{Changes in energy metabolism in ADMSCs}

We analyzed the effects of $3 \mathrm{D}$ and $2 \mathrm{D}$ culture on ADMSC energy metabolism (Fig. 6a). It is generally accepted that glycolysis and mitochondrial respiration are two major energy production pathways in cells [28, 29]. Through glycolysis, ADMSCs utilize glucose to generate 


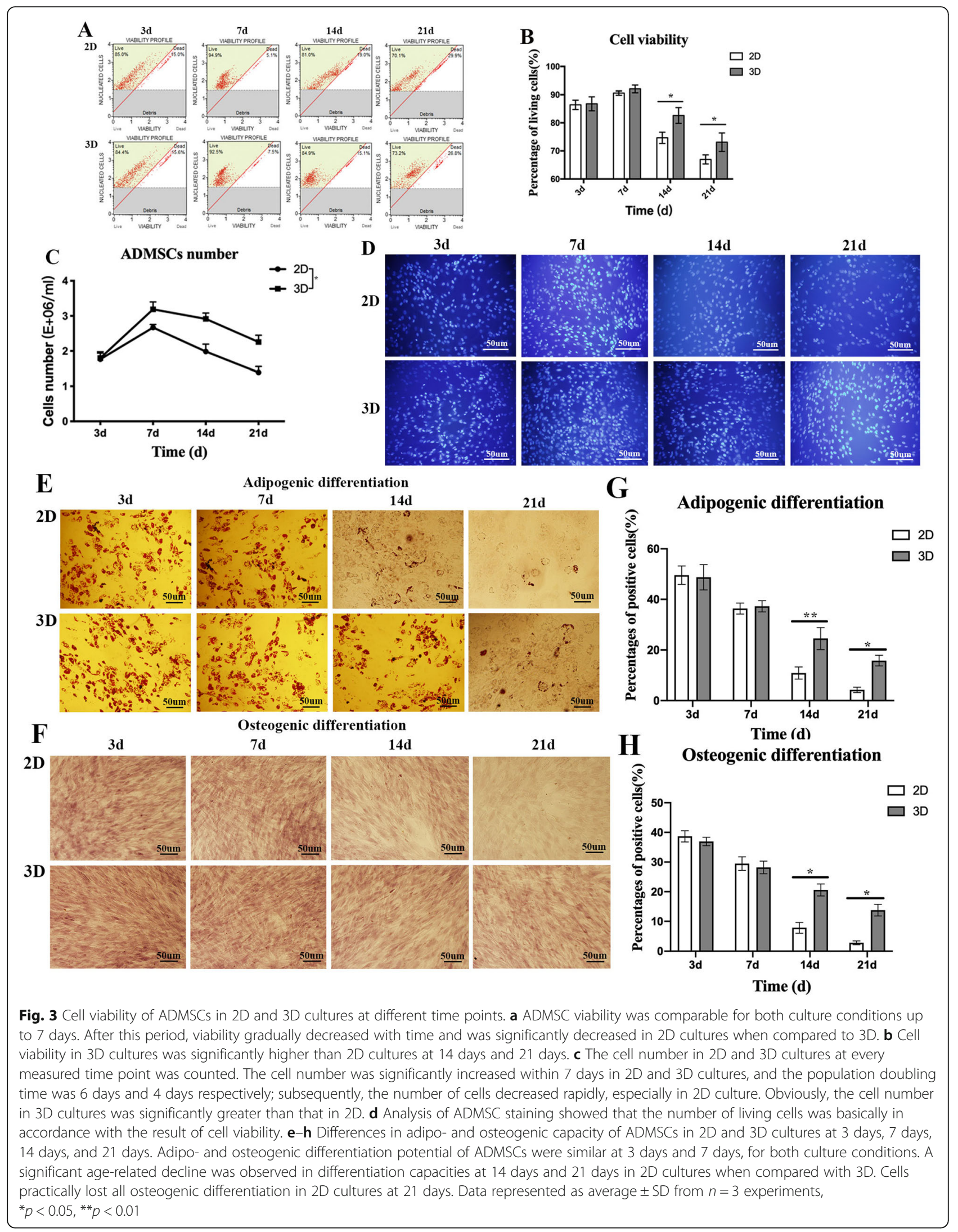



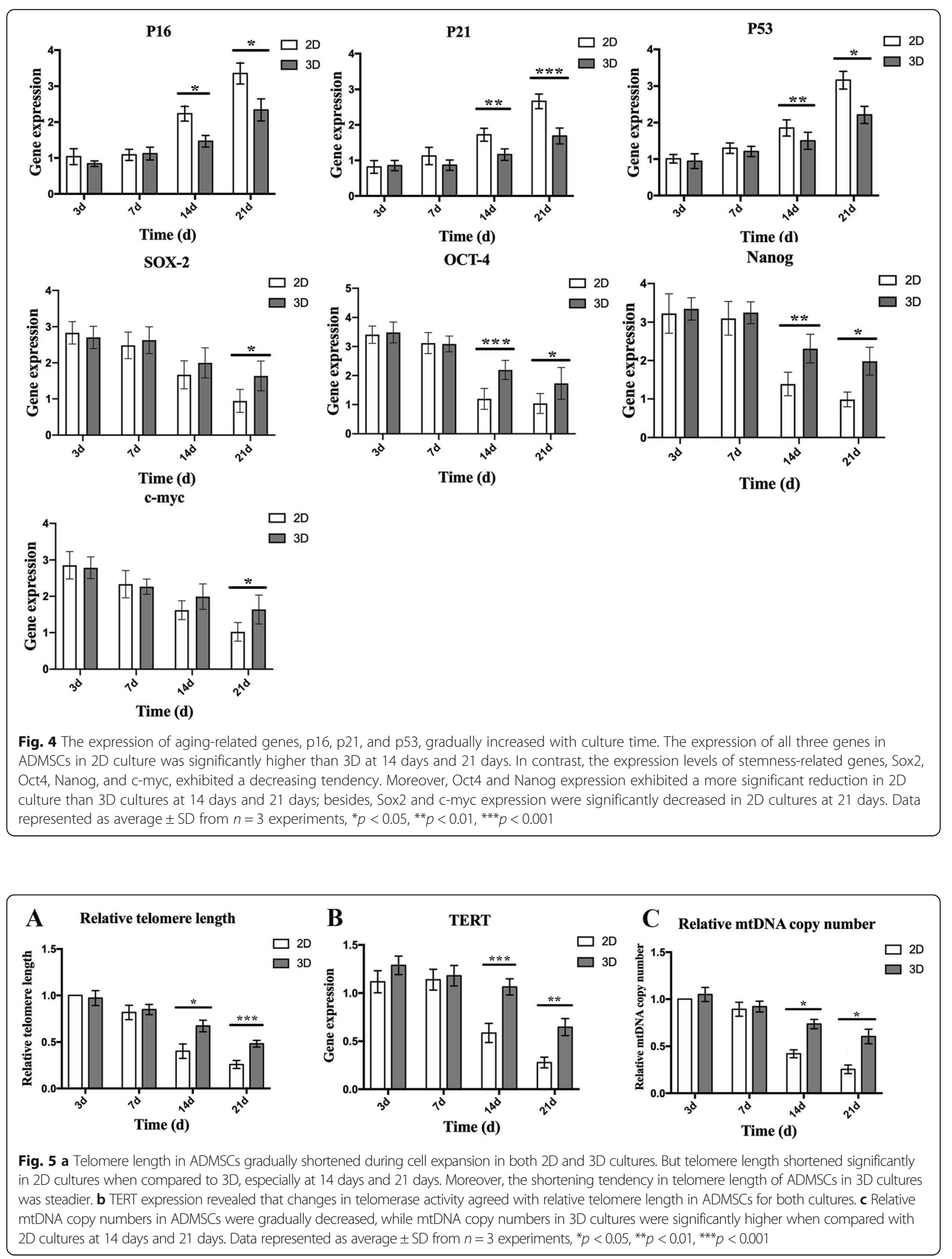
A

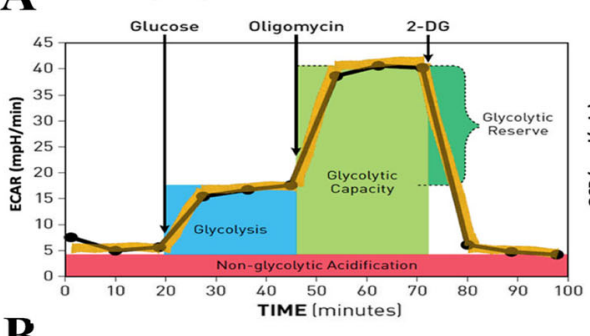

B
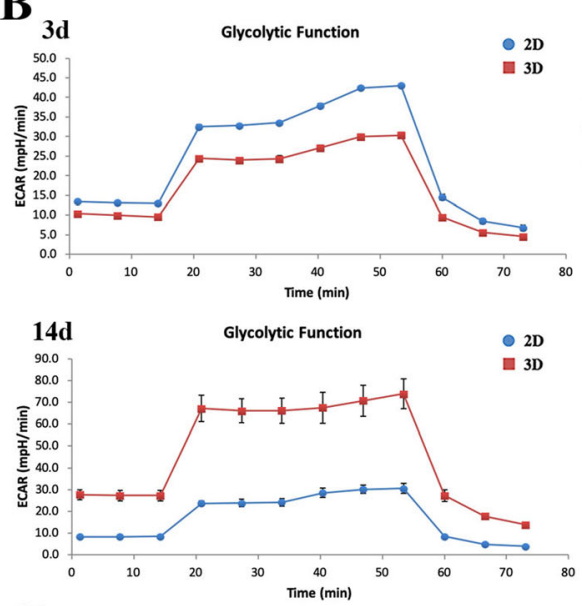

2D

C
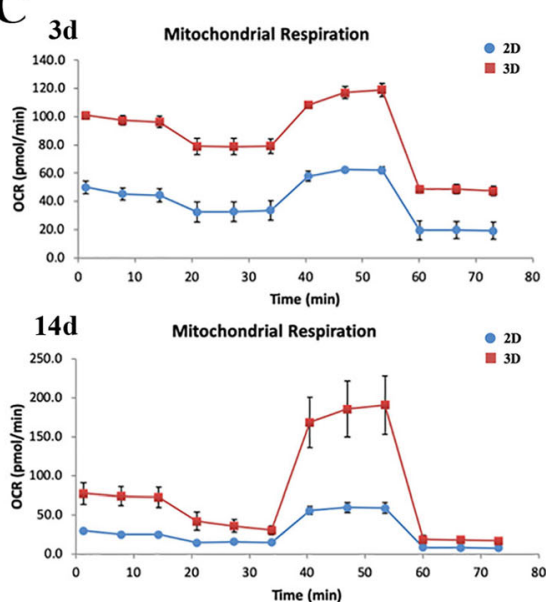

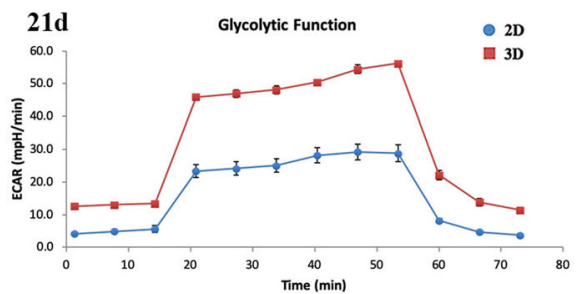

Mitochondrial Stress Test Profile
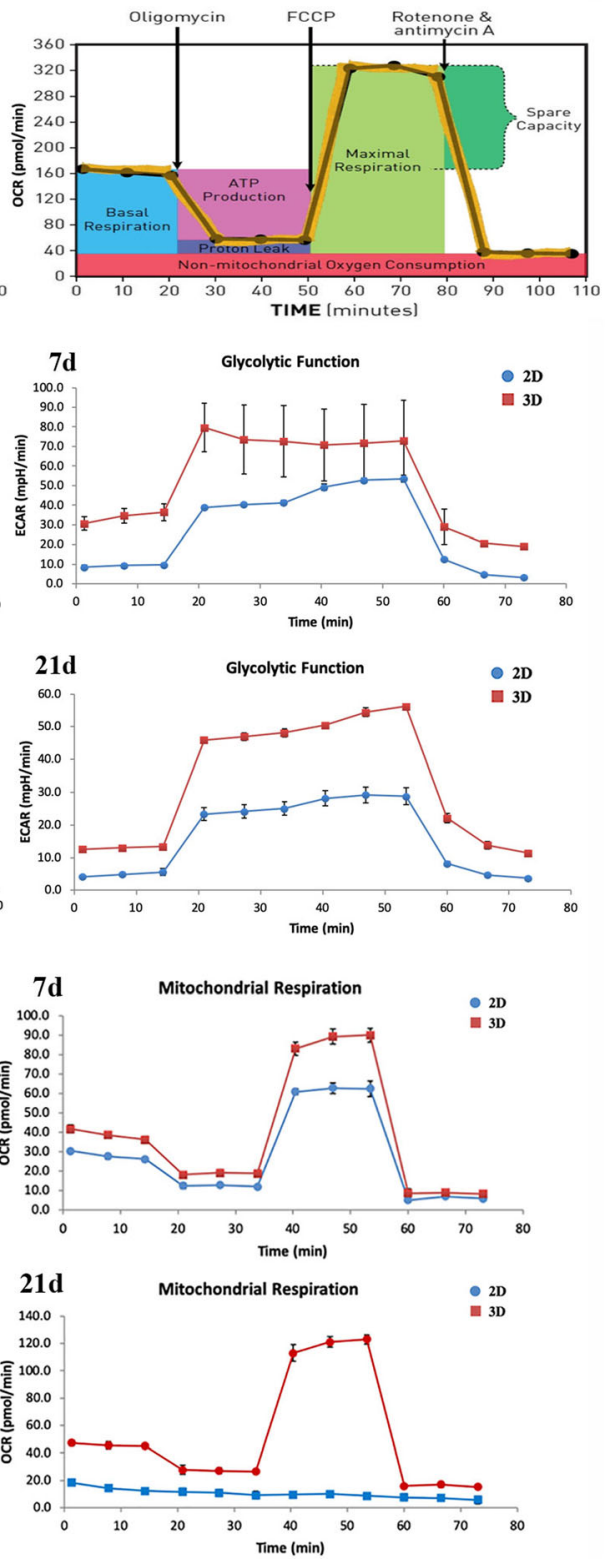

Fig. 6 Change in glycolysis and mitochondrial respiration levels in ADMSCs. a Glycolysis and mitochondrial stress tests. b ECAR levels in ADMSCS in 3D cultures were higher than 2D cultures, at 7 days, 14 days, and 21 days, but lower at 3 days. c OCR levels in ADMSCs in 3D cultures were higher when compared with 2D cultures at each time point. The experiment was repeated five times

lactate; thus, if cells are compromised, increased lactate levels could create an acidic environment, as assessed by ECAR [30], and increased OCR is an indicator of mitochondrial respiration. We observed that ECAR levels in ADMSCs in 3D cultures were higher than in 2D cultures at 7 days, 14 days, and 21 days (Fig. 6b), but slightly less at 3 days, as ECAR levels showed little differences between $2 \mathrm{D}$ and $3 \mathrm{D}$ cultures at this early stage. OCR levels of ADMSCs in 3D culture were higher when compared with 2D culture, at each time point (Fig. 6c). Taken together, the $3 \mathrm{D}$ culture of ADMSCs appeared to induce positive regulation of glycolysis and mitochondrial respiration, i.e., 3D cultures sustained energy metabolism stability in ADMSCs.

\section{Discussion}

Human ADMSCs are ideal candidates for diverse regenerative medicine approaches and tissue engineering strategies [31, 32]. However, it is interesting that therapies requiring ADMSCs often require ex vivo expansion approaches to generate the large numbers of cells required for patients and to overcome cell senescence 
limitations [6, 33]. It is widely recognized that 3D cultures create a pragmatic ECM and mimic in vivo development [20,34]. Therefore, 3D culture of MSCs could increase cell yields, enhance differentiation, maintain stemness, and provide promising strategies for MSC expansion on an industrial scale, with great potential for cell therapy and biotechnology [20, 22].

In terms of morphological and biochemical characteristics, in vitro MSC senescence is characterized by an enlarged flat cellular morphology; an increased SA- $\beta$-gal activity; an increased expression of p16, p21, and p53 [12, 35]; and a loss of stem cell properties [36]. Studies have reported that MSCs enter replicative senescence after extensive culture, leading to morphological and functional cellular changes [23, 37]. Cell senescence is a major factor that affects the proliferation and multi-lineage potential of MSCs [23, 38]. Here, we discovered that proliferation and adipogenic and osteogenic differentiation in ADMSCs steadily declined following extensive expansion, but notably, the decline in 2D culture was greater than 3D. In addition, 3D ADMSCs at different culture times were re-adhering in vessels, without hydrogel; we observed that these cells were morphologically younger when compared with $2 \mathrm{D}$ culture cells, at the same time period; and more importantly, SA- $\beta$-gal expression in these cells (3D) was lower. These observations agreed with the literature showing that significant changes in cell morphology were associated with increased SA- $\beta$-gal expression [11]. Such phenomenon has been rarely reported, but our data provides critical evidence that $3 \mathrm{D}$ culturing exerts positive effects on senescence-associated changes. Interestingly, recent evidence has indicated that even a short 3D culture of $72 \mathrm{~h}$ altered extensively expanded MSC characteristics [7].

Telomere length and telomerase activity represent cell senescence at the cellular level [39]. Moreover, cell lifespan is directly proportional to telomere length [40]. Thus, telomere shortening more than likely functions as a mechanism implicated in cellular senescence [39, 41]. Reports have identified a direct correlation between telomerase activity and stem cell function [37], with telomere attrition contributing to aging [42]. Furthermore, studies have demonstrated that the relative telomere length of ADMSCs declines with long-term passaging [38]. Our study suggests that telomere length and telomerase activity of ADMSCs decline with aging, but may be improved by $3 \mathrm{D}$ culture, further confirming that 3D culturing exerts positive effects in delaying senescence in ADMSCs.

Although MSC aging mechanisms during long-term expansion remain ill-defined, it has been shown that in addition to telomere shortening and telomerase activity reduction inducing senescence, oxygen free radical generation and altered mitochondrial function may have plausible roles in aging [43]. Additionally, previous studies have demonstrated that senescence is associated with metabolic changes in the oxidative state of the cell and that this process is linked to glycolytic ability and mitochondrial function [11-13], suggesting associations between changes in energy metabolism and senescence. The metabolic energy changes observed in ADMSCs (in 2D and 3D cultures) in this study reveal that ECAR and OCR levels vary greatly between dimensional cultures, over different cultivation periods. Overall, ECAR and OCR levels were higher in $3 \mathrm{D}$ cultures, suggesting that this approach had positive effects on glycolytic function and mitochondrial respiration in ADMSCs. Up to now, ADMSC energy metabolism research is in its infancy, and relationships between energy metabolism changes and ADMSCs senescence had rarely been reported. Accordingly, this study has preliminarily confirmed that $3 \mathrm{D}$ culturing slows ADMSC senescence, by improving mitochondrial function and energy metabolism.

Indeed, some reports have indicated a direct relationship between mitochondrial dysfunction and stem cell aging $[28,44]$. In several cell systems, mitochondrial dysfunction leads to respiratory chain dysfunction, which may be the result of mutation accumulation in mtDNA, in line with aging [45]. A critical mechanism underlying mitochondrial dysfunction is the expansion of mutations and deletions in mtDNA [29]. Additionally, these accumulated aging-related mutations and deletions lead to decreased mtDNA copy numbers [29, 45]. This study has shown that 3D culturing protects mtDNA in ADMSCs from aging-related impairments, suggesting that 3D cultures improve aging-related mitochondrial dysfunction.

Nevertheless, this study has some limitations. Firstly, some data did not reach statistical significance, which may be the result of lack of enough cell culture time in vitro. Similarly, we did not assess chondrogenic differentiation in ADMSCs, since the cells need to form spheroids during chondrogenic differentiation; the diversity in capability of sphere-forming of ADMSCs in 2D and 3D cultures has a great influence on chondrogenic differentiation; thus, it is difficult to evaluate chondrogenic differentiation ability of the ADMSCs. Finally, we revealed factors that impeded ADMSC growth in vitro, but an in-depth mechanistic exploration of factors that induce cellular senescence is required in future research.

\section{Conclusions}

Taken together, this study confirms that 3D culturing relieves senescence-related changes in ADMSCs and highlights the importance of developing 3D culture 
approaches for sustained and healthy MSC growth. There is no doubt that $3 \mathrm{D}$ culturing will contribute to effective ADMSC preparations for cellular therapy, and importantly, our findings lay the path for future ADMSC applications in biomedical research. However, further investigations will be required to fully evaluate the effectiveness and safety of ADMSCs in 3D culture for future in vivo therapies.

\section{Supplementary information}

Supplementary information accompanies this paper at https://doi.org/10. 1186/s13287-020-01744-1.

Additional file 1: Fig. S1. ADMSCs exhibit fibroblast-like, spindleshaped morphology, were spiral shaped and in alignment.

Additional file 2: Fig. S2. Flow cytometry analysis showing the presence of CD34 and CD45 negative (0.89\%) surface markers (a), and CD44 and CD105 positive (99.49\%) surface markers (b)

Additional file 3: Fig. S3. Model graph of 3D hydrogel culture.

\section{Abbreviations}

ADMSCs: Adipose-derived mesenchymal stem cells; 2D: Two-dimensional; MSCs: Mesenchymal stem cells; 3D: Three-dimensional; SA- $\beta$-gal: Senescenceassociated $\beta$-galactosidase; ECM: Extracellular microenvironment; RTqPCR: Real-time fluorescence quantitative polymerase chain reaction; gDNA: Genomic DNA; TERT: Telomerase reverse transcriptase; TERC: Telomerase RNA component; mtDNA: Mitochondrial DNA; ECAR: The extracellular acidification rate; OCR: Oxygen consumption rate

\section{Acknowledgements}

We thank International Science Editing (http://www.

internationalscienceediting. com) for editing this manuscript.

\section{Authors' contributions}

Qi-liang Yin and $\mathrm{Na}$ Xu: conception and design, collection and/or assembly of data, data analysis and interpretation, and manuscript writing. Hao-fan Jin and Wen-sen Liu: conception and design, supervision of the study, and final approval of manuscript. Dong-sheng Xu, Ming-xin Dong, Xiu-min Shi, and Yan Wang: design, collection and/or assembly of data, data analysis and interpretation, and statistical analysis; Zhuo Hao, Shuang-shuang Zhu, and Dong-hai Zhao: collection and/or assembly of data. All authors read and approved the final manuscript.

\section{Funding}

This research was supported by the Project Agreement for Science \& Technology Development, Jilin Province (no. 20190304027YY, no. $20200404135 Y$ Y) and by the Science and Technology Development Program of Jilin Provincial of China (no. 20190201148JC)

\section{Availability of data and materials}

The datasets used and/or analyzed during the current study available from the corresponding author on reasonable request.

\section{Ethics approval and consent to participate}

The use of human tissue was approved by the ethics committee of the First Hospital of Jilin University, China (EK Nr. 985/2016, 25 January 2018), and the donor gave written consent.

\section{Consent for publication}

Not applicable

\section{Competing interests}

The authors declare that they have no competing interests.

\section{Author details}

${ }^{1}$ Cancer Center at the First Hospital of Jilin University, 1 Xinmin Street, Changchun 130021, People's Republic of China. ${ }^{2}$ Jilin Medical University, Jilin 132013, People's Republic of China. ${ }^{3}$ Institute of Military Veterinary Medicine, Academy of Military Medical Sciences, Zoonosis Prevention and Control Key Laboratory, Changchun 130122, People's Republic of China.

Received: 24 February 2020 Revised: 18 May 2020

Accepted: 25 May 2020 Published online: 09 June 2020

\section{References}

1. Stoltz JF, de Isla N, Li YP, et al. Stem cells and regenerative medicine: myth or reality of the 21th century. Stem Cells Int. 2015;2015:734731.

2. Shingyochi $Y$, Orbay $H$, Mizuno $H$. Adipose-derived stem cells for wound repair and regeneration. Expert Opin Biol Ther. 2015;15:1285-92.

3. Luck J, Weil BD, Lowdell M, Mosahebi A. Adipose-Derived Stem Cells for Regenerative Wound Healing Applications: Understanding the Clinical and Regulatory Environment [published online ahead of print, 2019 Aug 13]. Aesthet Surg J. 2019;sjz214. https://doi.org/10.1093/asj/sjz214.

4. Mizuno H, Tobita M, Uysal AC. Concise review: adipose-derived stem cells as a novel tool for future regenerative medicine. Stem Cells. 2012;30:804-10.

5. Huang $H$, Kolibabka M, Eshwaran $R$, et al. Intravitreal injection of mesenchymal stem cells evokes retinal vascular damage in rats. FASEB J. 2019:33(12):14668-79.https://doi.org/10.1096/fj.201901500R.

6. Hoch Al, Leach JK. Concise review: optimizing expansion of bone marrow mesenchymal stem/stromal cells for clinical applications. Stem Cells Transl Med. 2015:4:412

7. Bartosh TJ, Ylostalo JH. Efficacy of 3D Culture Priming is Maintained in Human Mesenchymal Stem Cells after Extensive Expansion of the Cells. Cells. 2019:8(9):1031. Published 2019 Sep 5. https://doi.org/10.3390/ cells8091031.

8. Ravi M, Paramesh V, Kaviya SR, et al. 3D cell culture systems: advantages and applications. J Cell Physiol. 2015;230:16-26.

9. Dutta RC, Dutta AK. Cell-interactive 3D-scaffold; advances and applications. Biotechnol Adv. 2009:27:334-9.

10. Kouroupis D, Sanjurjo-Rodriguez C, Jones E, et al. Mesenchymal stem cell functionalization for enhanced therapeutic applications. Tissue Eng Part B Rev. 2019;25:55-77.

11. Stab BR 2nd, Martinez L, Grismaldo A, et al. Mitochondrial functional changes characterization in young and senescent human adipose derived MSCs. Front Aging Neurosci. 2016:8:299.

12. Fafian-Labora JA, Morente-Lopez M, Arufe MC. Effect of aging on behaviour of mesenchymal stem cells. World J Stem Cells. 2019;11:337-46.

13. Macrin D, Alghadeer A, Zhao YT, et al. Metabolism as an early predictor of DPSCs aging. Sci Rep. 2019;9:2195.

14. Khan H, Mafi $P$, Mafi R, et al. The effects of ageing on differentiation and characterisation of human mesenchymal stem cells. Curr Stem Cell Res Ther. 2018:13:378-83.

15. Sekiya I, Larson BL, Smith JR, et al. Expansion of human adult stem cells from bone marrow stroma: conditions that maximize the yields of early progenitors and evaluate their quality. Stem Cells. 2002;20:530-41.

16. Tachibana CY. Stem-cell culture moves to the third dimension. Nature. 2018; 558:329-31.

17. Liu Z, Tang M, Zhao J, et al. Looking into the future: toward advanced 3D biomaterials for stem-cell-based regenerative medicine. Adv Mater. 2018;30: e1705388.

18. Antoni D, Burckel H, Josset $\mathrm{E}$, et al. Three-dimensional cell culture: a breakthrough in vivo. Int J Mol Sci. 2015;16:5517-27.

19. Haycock JW. 3D cell culture: a review of current approaches and techniques. Methods Mol Biol. 2011;695:1-15.

20. Luo Y, Lou C, Zhang S, et al. Three-dimensional hydrogel culture conditions promote the differentiation of human induced pluripotent stem cells into hepatocytes. Cytotherapy. 2018;20:95-107.

21. Cesarz Z, Tamama K. Spheroid culture of mesenchymal stem cells. Stem Cells Int. 2016;2016:9176357.

22. Li Y, Guo G, Li L, et al. Three-dimensional spheroid culture of human umbilical cord mesenchymal stem cells promotes cell yield and stemness maintenance. Cell Tissue Res. 2015;360:297-307.

23. Truong NC, Bui KH, Van Pham P. Characterization of Senescence of Human Adipose-Derived Stem Cells After Long-Term Expansion. Adv Exp MedBiol. 2019;1084:109-28. https://doi.org/10.1007/5584_2018_235. 
24. Bae YJ, Kwon YR, Kim HJ, et al. Enhanced differentiation of mesenchymal stromal cells by three-dimensional culture and azacitidine. Blood Res. 2017; 52:18-24.

25. El-Hamid SA, Mogawer A. In vitro mesenchymal stem cells differentiation into hepatocyte-like cells in the presence and absence of 3D microenvironment. Comp Clin Pathol. 2013;23:1051-8.

26. Gutiérrez ML, Guevara JM, Echeverri OY, et al. Aggrecan catabolism during mesenchymal stromal cell in vitro chondrogenesis. Anim Cells Syst. 2013;17: 243-9.

27. Fajkus J, Sykorova E, Leitch AR. Telomeres in evolution and evolution of telomeres. Chromosom Res. 2005:13:469-79.

28. Bratic A, Larsson NG. The role of mitochondria in aging. J Clin Invest. 2013; 123:951-7.

29. Greaves LC, Nooteboom M, Elson JL, et al. Clonal expansion of early to midlife mitochondrial DNA point mutations drives mitochondrial dysfunction during human ageing. PLoS Genet. 2014;10:e1004620.

30. Zhang B, Wu J, Cai Y, et al. AAED1 modulates proliferation and glycolysis in gastric cancer. Oncol Rep. 2018;40:1156-64.

31. Choudhery MS, Badowski M, Muise A, et al. Donor age negatively impacts adipose tissue-derived mesenchymal stem cell expansion and differentiation. J Transl Med. 2014;12:8.

32. Jin HJ, Bae YK, Kim M, et al. Comparative analysis of human mesenchymal stem cells from bone marrow, adipose tissue, and umbilical cord blood as sources of cell therapy. Int J Mol Sci. 2013;14:17986-8001.

33. Stolzing A, Jones E, McGonagle D, et al. Age-related changes in human bone marrow-derived mesenchymal stem cells: consequences for cell therapies. Mech Ageing Dev. 2008;129:163-73.

34. Madl CM, LeSavage BL, Dewi RE, Lampe KJ, Heilshorn SC. Matrix Remodeling Enhances the Differentiation Capacity of Neural Progenitor Cells in3D Hydrogels. Adv Sci (Weinh). 2019;6(4):1801716. Published 2019 Jan 11. https://doi.org/10.1002/advs.201801716.

35. Zhang M, Du Y, Lu R, et al. Cholesterol retards senescence in bone marrow mesenchymal stem cells by modulating autophagy and ROS/p53/p21(Cip1/ Waf1) pathway. Oxidative Med Cell Longev. 2016;2016:7524308.

36. Tobita M, Tajima S, Mizuno H. Adipose tissue-derived mesenchymal stem cells and platelet-rich plasma: stem cell transplantation methods that enhance stemness. Stem Cell Res Ther. 2015:6:215.

37. Drela K, Stanaszek L, Nowakowski A, et al. Experimental strategies of mesenchymal stem cell propagation: adverse events and potential risk of functional changes. Stem Cells Int. 2019;2019:7012692.

38. Legzdina D, Romanauska A, Nikulshin S, et al. Characterization of senescence of culture-expanded human adipose-derived mesenchymal stem cells. Int J Stem Cells. 2016;9:124-36.

39. Saretzki G. Telomeres, telomerase and ageing. Subcell Biochem. 2018;90: 221-308.

40. Alrefaei Gl, Alkarim SA, Abduljabbar HS. Impact of Mothers' Age on Telomere Length and Human Telomerase Reverse Transcriptase Expression inHuman Fetal Membrane-Derived Mesenchymal Stem Cells [published correction appears in Stem Cells Dev. 2020 Mar 15;29(6):380-381]. Stem CellsDev. 2019;28(24):1632-45. https://doi.org/10.1089/scd.2019.0144.

41. Funayama R, Ishikawa F. Cellular senescence and chromatin structure. Chromosoma. 2007;116:431-40.

42. Yu KR, Kang KS. Aging-related genes in mesenchymal stem cells: a minireview. Gerontology. 2013;59:557-63.

43. Romano AD, Serviddio $G$, de Matthaeis $A$, et al. Oxidative stress and aging. Nephrol. 2010;23(Suppl 15):S29-36.

44. Fellous TG, Islam S, Tadrous PJ, et al. Locating the stem cell niche and tracing hepatocyte lineages in human liver. Hepatology. 2009;49:1655-63.

45. Miquel J, Economos AC, Fleming J, et al. Mitochondrial role in cell aging. Exp Gerontol. 1980;15:575-91.

\section{Publisher's Note}

Springer Nature remains neutral with regard to jurisdictional claims in published maps and institutional affiliations.

Ready to submit your research? Choose BMC and benefit from:

- fast, convenient online submission

- thorough peer review by experienced researchers in your field

- rapid publication on acceptance

- support for research data, including large and complex data types

- gold Open Access which fosters wider collaboration and increased citations

- maximum visibility for your research: over $100 \mathrm{M}$ website views per year

At $\mathrm{BMC}$, research is always in progress.

Learn more biomedcentral.com/submissions 